\title{
PARÂMETROS CARACTERÍSTICOS DA PULVERIZAÇÃO PNEUMÁTICA EM COPAS DE ÁRVORES DE EUCALIPTO ${ }^{1}$
}

\author{
Walter Luiz Castro Mewes ${ }^{2}$, Mauri Martins Teixeira ${ }^{3}$, Haroldo Carlos Fernandes ${ }^{3}$, José Cola Zanuncio ${ }^{4}$ e \\ Rafael Augusto Soares Tiburcio ${ }^{5}$
}

\begin{abstract}
RESUMO - Este trabalho foi desenvolvido com o objetivo de determinar os melhores parâmetros da pulverização utilizando pulverizador pneumático adaptado para a aplicação em florestas de eucalipto, através do estudo de gotas usando etiquetas hidrossensíveis. Essas etiquetas foram posicionadas nos dosséis dos eucaliptos em três alturas diferenciadas e três posições, com o auxílio do Feller-Buncher. A pulverização foi feita utilizando um pulverizador pneumático, em que se empregou uma vazão de ar de $11.300 \mathrm{~m}^{3} \mathrm{~h}^{-1}$ e TDP de $56,5 \mathrm{rad} \mathrm{s}^{-1}$ (540 rpm). Foram utilizados três índices volumétricos. Os parâmetros observados foram densidade de gotas, porcentagem de cobertura, diâmetro da mediana volumétrica (DMV), coeficiente de homogeneidade e amplitude relativa. Nas condições em que o experimento foi conduzido, o pulverizador pneumático utilizado atendeu às expectativas tanto em densidade de gotas quanto em porcentagem de cobertura, para a aplicação de inseticida em floresta de eucalipto. Os valores médios do coeficiente de homogeneidade e amplitude relativa foram condizentes com o tipo de pulverizador empregado. Assim, o pulverizador utilizado foi eficiente na aplicação de agrotóxicos em árvores adultas de eucalipto.
\end{abstract}

Palavras-chave: Pulverizador pneumático; Etiquetas hidrossensíveis; Controle de pragas.

\section{CHARACTERISTIC PARAMETERS OF AIR SPRAYING IN EUCALYPTUS TREE CANOPY}

\begin{abstract}
This work was developed with the objective of determining the best parameters of spraying using pneumatic spray adapted for application in eucalyptus forests, through the study of droplets using water sensitive paper. These papers were placed in the canopies of the eucalyptus trees in three heights and three different positions in relation to the canopy, with the assistance of Feller-Buncher. The spraying was done using a pneumatic sprayer with an air flow of 11,300 $\mathrm{m}^{3} \mathrm{~h}^{-1}$ and PTO of $56.5 \mathrm{rad} \mathrm{s}^{-1}$ (540 rpm). Three Volumetric indexes were used. The parameters measured were droplet density, percentage of coverage, Volume median diameter (VMD), Homogeneity Coefficient and Relative Amplitude. In the conditions in which the experiment was conducted, the pneumatic sprayer met the expectations of droplet density and percentage of coverage for insecticide application in eucalyptus forest. The average values of the coefficient of homogeneity and relative amplitude were in agreement with the type of sprayer. So the spray used was efficient in the application of pesticides in adult trees of eucalyptus.
\end{abstract}

Keywords: Pneumatic spray; Water sensitive paper; Pest control.

\footnotetext{
${ }^{1}$ Recebido em 24.09.2012 aceito para publicação em 07.05.2015.

${ }^{2}$ Universidade Federal de Viçosa, Reitoria, Central de Ensino e Desenvolvimento Agrário de Florestal, Florestal, MG - Brasil. E-mail: <mewes@ufv.br>.

${ }^{3}$ Universidade Federal de Viçosa, Centro de Ciências Agrárias, Departamento de Engenharia Agrícola, Campus da UFV, Vicosa, MG - Brasil.E-mail: <mauri@ufv.br>e<haroldo@ufv.br>.

${ }^{4}$ Universidade Federal de Viçosa, Centro de Ciências Biológicas e da Saúde, Vicosa, MG - Brasil. E-mail: <zanuncio@ufv.br>.

${ }^{5}$ Universidade Federal de Viçosa, Centro de Ciências Agrárias, Programa de Pós-Graduação em Engenharia Agrícola, Campus da UFV, Vicosa, MG - Brasil. E-mail: <rastiburcio@yahoo.com.br>.
}

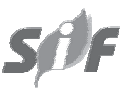

Revista Árvore, Viçosa-MG, v.39, n.4, p.635-640, 2015 http://dx.doi.org/10.1590/0100-67622015000400005 


\section{INTRODUÇ̃̃O}

Entre as várias etapas da mecanização florestal, a aplicação de fitossanitários merece atenção especial, pois atuam no controle de pragas, doenças e plantas daninhas, evitando perdas de produtividade no plantio de eucalipto. As espécies do gênero Eucalyptus são danificadas por insetos de hospedeiros nativos da flora brasileira, especialmente da família Myrtaceae (ZANUNCIO, 2001 apud PEREIRA, 2008). Entre os desfolhadores, destaca-se Thyrinteina arnobia (Lepidoptera: Geometridae) como o mais daninho dessa ordem para a eucaliptocultura nacional (HOLTZ et al., 2003).

Nas infestações por insetos-praga, o tratamento pode ser feito através do controle biológico, físico ou químico, porém, quando as infestações ocorrem nas copas das árvores que ultrapassam $15 \mathrm{~m}$ de altura e se localizam em pontos específicos da floresta, o controle torna-se difícil e caro. O método de controle mais utilizado é pela pulverização, que consiste na fragmentação do líquido em gotas nos pulverizadores. Na pulverização devem ser levados em consideração diversos fatores, como o momento certo da aplicação; o uso do produto adequado, responsável pela eficácia do controle; e o uso da máquina correta, em que se tenham alta eficiência da pulverização e a correta deposição de gotas sobre o alvo (MEWES et al., 2011).

A pulverização pneumática ocorre com a injeção da calda sob baixa pressão em uma corrente de ar, fragmentando o líquido em gotas e conduzindo-as até o alvo. As gotas pequenas resultantes da pulverização pneumática podem ser afetadas pela própria corrente de ar responsável pelo seu transporte até o alvo e pelas condições climáticas, causando deriva e evaporação. No entanto, a penetração de gotas maiores no interior da copa das árvores pode ser reduzida devido ao impedimento causado pelas folhas e pelo atrito com o ar (MEWES et al., 2011).

Na aplicação de agrotóxicos em plantas arbóreas é comum, devido à alta densidade foliar, que grande parte das gotas não atinja o interior do dossel. Nessa situação, a uniformidade de distribuição de líquido no dossel da planta fica prejudicada, causando baixa eficácia de controle. Devido a essa baixa eficácia, os produtores aumentam o volume de calda utilizado e a dose do agrotóxico, ocasionando aumento no custo de produção e contaminação ambiental (PAI et al., 2008).
Obter uniformidade de distribuição no dossel de plantas arbóreas sempre foi um dos objetivos de pesquisadores e produtores. A preocupação com a sustentabilidade, em que o uso de defensivos seja usado com cautela, tornou-se realidade em vários países. A maneira de praticar agricultura sustentável, sem agredir o meio ambiente, é aplicar o fitossanitário somente quando for preciso, em quantidade necessária e em local necessário (FAROOQ; LANDERS, 2004).

Um dos métodos de avaliação da qualidade da aplicação de agrotóxicos é o uso de papel sensível à água. Esses papéis apresentam, em uma de suas faces, a presença da substância bromofenol. Em contato com a água, ocorre a ionização dessa substância, ficando o local do contato com coloração azul. Essa coloração contrasta com o amarelo, que é a cor de fundo do papel, permitindo, assim, a caracterização do tamanho e formato das gotas depositadas na etiqueta. O conhecimento do espectro das gotas que atingem o alvo permite adequar o tamanho das gotas às condições locais, garantindo, assim, aplicação eficiente de produtos fitossanitários (CUNHA, 2003).

A utilização de papel hidrossensível em árvores para avaliar a distribuição do líquido no dossel por análise de imagem é descrita por Moor et al. (2000). Segundo esses autores, esse método de calibração de pulverizadores elimina o erro humano, mantendo um padrão de qualidade e oferecendo análise rápida e fácil da real condição da pulverização.

Cross et al. (2001), testando a distribuição da calda e as perdas causadas por deriva em árvores frutíferas utilizando papel hidrossensível e análise de imagens, descreveram a técnica como rápida e eficaz.

Diferentes volumes de aplicação e sua influência na qualidade da pulverização de laranjeiras, utilizando etiquetas hidrossensíveis, são descritos como capazes de diferenciar os tratamentos estudados (SALYANI; FOX, 1999).

A durabilidade de etiquetas amostradas por longo período não altera o tamanho, cor e forma das gotas quando armazenadas em condições adequadas (SALYANI, 1999).

Marti et al. (2006), avaliando a otimização da tecnologia de aplicação de fitossanitários em plantas arbóreas, concluíram que não é fácil conseguir distribuição uniforme sobre o dossel da planta. Afirmaram que 
aumentar a penetração de gotas é um objetivo importante para o desenvolvimento dos pulverizadores hidropneumáticos e para a economia de inseticidas.

O objetivo deste trabalho foi avaliar os parâmetros da pulverização utilizando pulverizador pneumático na aplicação em florestas de eucalipto, através do estudo de gotas com o uso de etiquetas hidrossensíveis, visando à escolha de melhores parâmetros para pulverização.

\section{MATERIAL E MÉTODOS}

Os experimentos foram conduzidos em área de reflorestamento de eucalipto (Eucalyptus grandis) da Celulose Nipo-Brasileira (Cenibra), no Município de Belo Oriente, MG. Os eucaliptos pertenciam a um talhão de 43,16 ha, plantado com o clone tipo $111 \mathrm{C} 89$, com idade de 6,3 anos.

Os experimentos foram instalados no delineamento em blocos casualizados com três repetições e esquema de parcelas subsubdivididas, tendo nas parcelas os volumes de aplicação nas subparcelas e as alturas e nas subsubparcelas, as posições. Cada árvore representou uma parcela.

O volume de aplicação foi determinado através da metodologia do "Tree Row Volume (TRV)" e dos índices volumétricos preestabelecidos.

O TRV foi determinado através da equação 1 , em que o TRV é o volume dos dosséis das plantas (m3 ha-1); H, a altura do dossel (m); L, o diâmetro do dossel (m); e D, a distância entre linhas de plantio (m).

$$
T R V=\frac{H \cdot L .10000}{D}
$$

Os índices volumétricos utilizados foram de $27,0 \mathrm{~mL} \mathrm{~m}^{-3}$ de copa (Índice 1), 20,2 $\mathrm{mL} \mathrm{m}^{-3}$ de copa (Índice 2) e 12,8 $\mathrm{mL} \mathrm{m}^{-3}$ de copa (Índice 3). Para isso, utilizaram-se as três primeiras velocidades do trator, obtendo, respectivamente, 0,$46 ; 0,66$; e $0,96 \mathrm{~m} \mathrm{~s}^{-1}$, com uma vazão de $24,1 \mathrm{~L} \mathrm{~min}^{-1}$.

Com base no TRV médio de copa e no índice volumétrico, foram determinados os volumes de aplicação.

Colocaram-se etiquetas de papel hidrossensíveis posicionadas nos dosséis dos eucaliptos, em três alturas diferenciadas (terços inferior, médio e superior do dossel) e três posições em relação à copa (frente, lateral e oposta à pulverização). Para o devido posicionamento dessas etiquetas, foi utilizada uma máquina florestal denominada Feller-Buncher, cortando-se a árvore a ser pulverizada e conduzindo-a, pelo seu cabeçote, até próximo do chão, quando as etiquetas eram presas nas referidas posições, retornando, assim, a árvore para a sua posição original e a mantendo em pé até que a aplicação fosse realizada.

A pulverização foi feita utilizando um pulverizador pneumático, marca Jacto, acoplado a um trator da marca New Holland, modelo TL85E. Empregou-se uma vazão de ar de $11.300 \mathrm{~m}^{3} \mathrm{~h}^{-1}$, utilizando $56,5 \mathrm{rad} \mathrm{s}^{-1}$ (540 rpm) no eixo da tomada de potência. Durante a coleta de dados, as condições do vento, a pressão barométrica, a temperatura e a umidade relativa foram monitoradas através de, respectivamente, anemômetro, barômetro, termômetro e higrômetro digitais previamente calibrados.

Após a pulverização, a copa da árvore era novamente abaixada pelo Feller-Buncher até próximo do solo, sendo as etiquetas retiradas, acondicionadas em sacos de papel devidamente separados de acordo com sua altura e posição de colocação na copa e levadas ao laboratório. Essas etiquetas foram digitalizadas, utilizando scanner HP, modelo Scanjet 3770, a uma resolução de 1.200 dpi, e analisadas com o auxílio do software IMAGE TOOL, versão 3.0, conforme também realizado por Rodrigues (2005), Ruas (2007) e Magno Júnior (2008).

Para determinação do tamanho das gotas, considerou-se o fator de espalhamento, segundo a equação 2 proposta por Chaim et al. (1999), em que F é o fator de espalhamento e $\mathrm{D}$, o diâmetro-limite de cada classe de tamanho da mancha $(\mathrm{mm})$.

$F=0,74057+0,0001010399 \quad D+0,2024884 \ln (D)(2)$

A avaliação da deposição foi realizada considerando os parâmetros porcentagem de cobertura, densidade de gotas, diâmetro da mediana volumétrica (DMV), coeficiente de homogeneidade e amplitude relativa. Os dados foram submetidos à análise de variância e as médias, comparadas utilizando os testes F e Tukey, ambos a $5 \%$ de probabilidade.

\section{RESULTADOS}

Durante a aplicação, a temperatura variou entre $24^{\circ} \mathrm{C}$ e $28^{\circ} \mathrm{C}$ e o vento próximo ao solo, entre 0,0 e $1,9 \mathrm{~m} \mathrm{~s}^{-1}$, sendo a pressão barométrica de $1.002 \mathrm{mbar}$ e a umidade relativa, acima de $62 \%$. Com base no TRV médio de $102.228 \mathrm{~m}^{3} \mathrm{ha}^{-1}$ de copa, foram determinados os volumes de aplicação, conforme a Tabela 1 .

Revista Árvore, Viçosa-MG, v.39, n.4, p.635-640, 2015 
Tabela 1 - Índice volumétrico e volume de pulverização aplicado no ensaio.

Table 1 - Volumetric index and volume of spray applied to the test.

\begin{tabular}{ccc}
\hline Tratamentos & Índice volumétrico $\left(\mathrm{mL} \mathrm{m}^{-3}\right.$ de Dossel $)$ & Volume de aplicação $\left(\mathrm{L} \mathrm{ha}{ }^{-1}\right)$ \\
\hline IV 1 & 27,0 & 2.760 \\
IV 2 & 20,2 & 2.065 \\
IV 3 & 12,8 & 1.308 \\
\hline
\end{tabular}

$\mathrm{Na}$ análise de variância da densidade de gotas em função do índice volumétrico aplicado, altura das etiquetas na planta e posição destas no dossel, somente a altura apresentou diferença significativa nas médias a $5 \%$ de probabilidade. No teste de Tukey das médias da densidade de gotas em diferentes alturas do dossel foi observado o maior valor no terço inferior do dossel, sendo estatisticamente diferente dos encontrados nos terços médio e superior, não tendo estes últimos diferença significativa entre suas médias (Tabela 2).

$\mathrm{Na}$ análise de variância da porcentagem de cobertura da etiqueta em função do índice volumétrico aplicado, altura das etiquetas na planta e posição destas no dossel, somente a variável posição e a interação altura $x$ volume apresentaram diferença significativa nas médias a $5 \%$ de probabilidade. $\mathrm{Na}$ Tabela 3 são apresentados os resultados do teste de Tukey dos valores de posição. Uma porcentagem de cobertura maior nas partes frontal e interna do dossel em relação à posterior era esperada em função da posição do pulverizador, entretanto essa diferença não foi significativa. Na Tabela 4 são apresentadas as médias da porcentagem de cobertura em razão dos índices volumétricos aplicados.

Na análise de variância do DMV em razão do índice volumétrico aplicado, altura das etiquetas na planta e posição destas no dossel não houve efeito significativo a $5 \%$ de probabilidade entre as variáveis estudadas nem entre as interações. Os valores médios do DMV nas diferentes alturas das copas variaram de $345 \mu \mathrm{m}$ a $465 \mu \mathrm{m}$. O maior valor foi encontrado no terço médio, mas, apesar de ser um valor mais alto, não diferiu estatisticamente daqueles verificados nos terços superior e inferior.

O coeficiente de homogeneidade $(\mathrm{CH})$ consiste na relação entre o diâmetro da mediana volumétrica e o diâmetro da mediana numérica e foi avaliado para fornecer referência com relação à dispersão dos diâmetros das gotas que atingiram o alvo. $\mathrm{Na}$ análise
Tabela 2 - Densidade de gotas em relação à altura do dossel na pulverização com vazão de ar de $11.300 \mathrm{~m}^{3}$ $\mathrm{h}^{-1}$ e TDP a $56,5 \mathrm{rad} \mathrm{s}^{-1}$

Table 2 - Density of droplets in relation to canopy height in the spray with air flow of $11,300 \mathrm{~m}^{3} \mathrm{~h}^{-1}$ and PTO to $56.5 \mathrm{rad} \mathrm{s}^{-1}$.

\begin{tabular}{lc}
\hline Altura do dossel & Densidade de gotas $\left(\right.$ Gotas $\left.\mathrm{cm}^{-2}\right)$ \\
\hline 1- Terço inferior & $129,7 \mathrm{~A}$ \\
2- Terço médio & $91,5 \mathrm{~B}$ \\
3- Terço superior & $113,71 \mathrm{~B}$ \\
\hline
\end{tabular}

Os valores seguidos pela mesma letra maiúscula não diferem a $5 \%$ de probabilidade, pelo teste de Tukey.

Values followed by the same capital letter do not differ at 5\% of probability, by Tukey test.

Tabela 3 - Porcentagem de cobertura nas posições do dossel na pulverização com vazão de ar de $11.300 \mathrm{~m}^{3} \mathrm{~h}^{-1}$ e TDP a $56,5 \mathrm{rad} \mathrm{s}^{-1}$

Table 3 -Percentage of coverage in the positions of the canopy in the spray with air flow of $11,300 \mathrm{~m}^{3} \mathrm{~h}^{-1}$ and PTO to $56.5 \mathrm{rad} \mathrm{s}^{-1}$

\begin{tabular}{cc}
\hline Posição no dossel & Cobertura (\%) \\
\hline Frontal & $18,8^{\mathrm{A}}$ \\
Interna & $18,8^{\mathrm{A}}$ \\
Posterior & $13,5^{\mathrm{A}}$ \\
\hline
\end{tabular}

Os valores seguidos pela mesma letra maiúscula não diferem a 5\% de probabilidade, pelo teste de Tukey.

Values followed by the same capital letter do not differ at $5 \%$ of probability, by Tukey test.

de variância do Coeficiente de Homogeneidade em função do índice volumétrico aplicado, altura das etiquetas na planta e posição destas no dossel, somente a variável altura teve diferenças estatísticas significativas entre as médias. A Tabela 5 apresenta o teste de média do coeficiente de homogeneidade. O maior valor foi no terço médio do dossel, porém não foi significativamente diferente pelo teste de Tukey a $5 \%$ de probabilidade.

A amplitude relativa (AR) é mais uma referência no estudo da variação dos diâmetros das gotas pulverizadas. Os valores variam de 0,8 a 1,2. Na análise de variância da amplitude relativa, não houve diferenças estatísticas significativas a $5 \%$ de probabilidade, em nenhuma variável ou interação. 
Tabela 4 - Porcentagem de cobertura de cada índice volumétrico em função da altura do dossel na pulverização com vazão de ar de $11.300 \mathrm{~m}^{3} \mathrm{~h}^{-1}$ e TDP a $56,5 \mathrm{rad} \mathrm{s}^{-1}$.

Table 4 - Percentage of coverage for each volumetric index as a function of canopy height in the spray with air flow of $11,300 \mathrm{~m}^{3} \mathrm{~h}^{-1}$ and PTO to $56.5 \mathrm{rad} \mathrm{s}^{-1}$.

\begin{tabular}{cccc}
\hline \multicolumn{3}{c}{ Altura do dossel } \\
\hline Índice volumétrico $\left(\mathrm{mL} \mathrm{m}^{-3}\right)$ & Terço médio & Terço superior \\
\hline 27,0 & $21,4^{\mathrm{A}}$ & $15,0^{\mathrm{AB}}$ & $9,50^{\mathrm{B}}$ \\
20,2 & $8,2^{\mathrm{B}}$ & $21,7^{\mathrm{A}}$ & $21,0^{\mathrm{A}}$ \\
12,8 & $20,0^{\mathrm{A}}$ & $18,9^{\mathrm{A}}$ & $17,5^{\mathrm{A}}$ \\
\hline
\end{tabular}

Os valores seguidos pela mesma letra maiúscula não diferem a 5\% de probabilidade, pelo teste de Tukey.

Values followed by the same capital letter do not differ at 5\% of probability, by Tukey test.

Tabela 5 - Coeficiente de homogeneidade em função da altura na pulverização com vazão de ar de $11.300 \mathrm{~m}^{3} \mathrm{~h}^{-1} \mathrm{e}$ TDP a $56,5 \mathrm{rad} \mathrm{s}^{-1}$.

Table 5 - Coefficient of homogeneity as a function of height in the spray with air flow of $11,300 \mathrm{~m}^{3} \mathrm{~h}^{-1}$ and PTO to $56.5 \mathrm{rad} \mathrm{s}^{-1}$.

\begin{tabular}{lc}
\hline Altura no dossel & $\mathrm{CH}$ \\
\hline Terço inferior & $3,3841^{\mathrm{A}}$ \\
Terço médio & $5,7007^{\mathrm{A}}$ \\
Terço superior & $3,4619^{\mathrm{A}}$ \\
\hline
\end{tabular}

Os valores seguidos pela mesma letra maiúscula não diferem a $5 \%$ de probabilidade, pelo teste de Tukey.

Values followed by the same capital letter do not differ at 5\% of probability, by Tukey test.

\section{DISCUSSÃO}

Em relação à densidade de gotas, como a pulverização foi feita de baixo para cima, era esperada maior densidade de gotas no terço inferior do dossel. O "efeito parede" fez que as próprias folhas servissem de barreira para a penetração do líquido no interior do dossel. Os valores médios obtidos de densidade de gotas variaram de 91 a 129 gotas $\mathrm{cm}^{-2}$. Assim, em todas as alturas estudadas, o número de gotas foi suficiente para uma aplicação de inseticida (MEWES et al., 2011).

O maior número de gotas no terço inferior é importante no controle da lagarta, uma vez que elas preferem as folhas mais velhas para se alimentarem, e essas folhas se encontram nessa região do dossel (SEPCHT et al., 2006). As diferenças estatísticas entre os terços inferiores e os terços médios podem ser justificadas pela arquitetura da planta. Isso porque a área estudada era uma floresta de clones, e estes tendiam a ter forma semelhante.

A porcentagem de cobertura foi semelhante nas três alturas em cada um dos índices volumétricos estudados, à exceção do terço superior do índice volumétrico 1 e do terço inferior do índice volumétrico 2, o que pode ser justificado pelo alto coeficiente de variação do experimento (50,46\%). Esse alto coeficiente de variação na porcentagem de cobertura foi justificado pelo tipo de pulverizador usado, que não teve boa uniformidade no tamanho de gotas, o que também afetou o DMV. Outros fatores que contribuíram para isso foram a longa distância entre o pulverizador e o alvo e a alta velocidade de lançamento das gotas, provocando manchas irregulares nas etiquetas hidrossensíveis no sentido do fluxo de ar, o que tirou a esfericidade da gota.

Em relação ao DMV, são valores esperados, uma vez que o pulverizador pneumático não usa um bico pulverizador para fracionar a calda e gerar a gota e, sim, a corrente de ar produzida pelo ventilador, e esse tipo de fracionamento é menos eficiente que as pontas pulverizadoras. Com maior volume para fracionar, há tendência de as gotas serem maiores e em maior número, e isso eleva o valor de DMV.

Em relação ao coeficiente de homogeneidade, uma das possíveis causas desse valor alto no terço médio pode estar relacionada com a arquitetura do dossel. Por serem clones, possuem tendência a apresentar formato semelhante entre as plantas, e isso pode ter influenciado os resultados.

Os valores de AR variaram de 0,8 a 1,2. O valor ideal do AR foi zero, em que teriam todas as gotas com o mesmo diâmetro. O pulverizador pneumático tem variação maior de gotas, portanto o AR próximo a 1 está adequado a esse tipo de equipamento.

\section{CONCLUSÃO}

Nas condições em que o experimento foi conduzido, conclui-se que o pulverizador pneumático utilizado atendeu às expectativas tanto de densidade de gotas

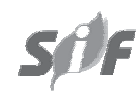

Revista Árvore, Viçosa-MG, v.39, n.4, p.635-640, 2015 
quanto de porcentagem de cobertura, para a aplicação de inseticida em floresta de eucalipto. Os valores médios do coeficiente de homogeneidade e amplitude relativa foram condizentes com o tipo de pulverizador empregado. Assim, o pulverizador utilizado foi eficiente na aplicação de agrotóxicos em árvores adultas de eucalipto.

\section{REFERÊNCIAS}

CHAIM, A.; MAIA, A.H.N.; PESSOA, M.C.P.Y. Estimativa da deposição de agrotóxicos por análise de gotas. Pesquisa Agropecuária Brasileira, v.34, n.6, p.963-969, 1999.

CROSS, J.V.; WALKLATE, P.J.; MURRAY, R.A.; RICHARDSON, G.M. Spray deposits and losses in different sized apple trees from an axial fan orchard sprayer. Effects of spray liquid flow rate. Crop Protection, v.20, n.1,p.13-30, 2001.

CUNHA, J.P.A.R. Tecnologia de aplicação convencional de fungicida de contato no controle de doenças do feijoeiro. 2003. 79f. Tese (Doutorado em Engenharia Agrícola) - Universidade Federal de Viçosa, Viçosa, MG, 2003.

FAROOQ, M.; LANDERS, A.J. Interactive effects of air, liquid and canopies on spray patterns of axial-flow sprayers. St. Joseph, MI: ASABE, 2004. (Paper, 1001).

HOLTZ, A. M; ZANUNCIO, J. C.; OLIVEIRA, H. G.; PALLINI, A.; MARINHO, J. S.; OLIVEIRA, C. L.; PINON, T. B. M. Aspectos biológicos de Thyrinteina arnobia (Lep.: Geometriadae) provenientes de lagartas criadas em folhas de Eucalyptus cloeziana ou de Psidium guajava sob condições de campo. Revista Árvore, v.27, n.6, p.897-901, 2003.

MAGNO JÚNIOR, R.G. Desenvolvimento de sistema eletrônico para pulverização e atração de gotas em plantas cítricas. 2008. 69f. Dissertação (Mestrado em Engenharia Agrícola) - Universidade Federal de Viçosa, Viçosa, MG, 2008.

MEWES, W. L. C.; TEIXEIRA, M. M.; FERNANDES, H. C.; CECON, P. R.; ALVARENGA,
C. B. Deposiçãp de calda em eucalipto, utilizando pulverização pneumática. Bioscience Journal, v.27, n.2, p.283-288, 2011.

MARTI, S.P.; POLO, J.R.R.; MOYA, E.G.; MONTEROLA, L.V.; ESCOLA, A. Optimizing pesticide spray application in tree crops. St. Joseph, MI: ASABE, 2006. (Paper, 1128).

MOOR, B.A.; LANGENAKENS, J.; VEREECKE, E. Image analysis of water sensitive paper as a tool for the evaluation of spray distribution of orchard sprayers: aspects of applied biology. Aspects of Applied Biology, v.57, p.329-342, 2000.

PAI, N.; SALYANI, M.; SWEEB, R.D. Adjusting airblast sprayer airflow based on tree foliage density. St. Joseph, MI: ASABE, 2008. (Paper, 3653).

PEREIRA, F. F.; ZANUNCIO, A. J. V.; FELIPE, J. P. M.; LORENZON, A. S.; CANEVARI, G. C. Desenvolvimento e reprodução de Dirphia moderata (Lepidoptera: Satutniidae) em Eucalyptus cloeziana e Psidium guajava em laboratório. Revista Árvore, v.32, n.6, p.1119-1124, 2008.

RODRIGUES, G.J. Critérios rastreáveis na aplicação de inseticida no controle do bicho mineiro do cafeeiro. 2005. $118 \mathrm{f}$. Tese (Doutorado em Engenharia Agrícola) Universidade Federal de Viçosa, Viçosa, MG, 2005.

RUAS, R.A.A. Tecnologia de aplicação de glyphosate para certificação de produtos agrícolas. 2007. 107f. Tese (Doutorado em Engenharia Agrícola) Universidade Federal de Viçosa, Viçosa, MG, 2007.

SALYANI, M. A technique for stabilizing droplet spots on oil-sensitive paper Transactions of the ASAE, v.42, n. 1, p.45-48, 1999.

SALYANI, M.; FOX, R.D. Evaluation of spray quality by oil and water-sensitive papers. Transactions of the ASAE, v.42, n.1, p.37-43, 1999 .

SPECHT, A.; FORMENTINI, A.C.; CORSEUIL, E. Biologia de Hylesia nigricans (Berg) (Lepidoptera, Saturniidae, Hemileucinae). Revista Brasileira de Zoologia, v.23, n.1, p.248-255, 2006. 